\title{
Pediatric Quality of Life Inventory
}

National Cancer Institute

\section{Source}

National Cancer Institute. Pediatric Quality of Life Inventory. NCI Thesaurus. Code C78652.

A parent report or patient self report for measuring health-related quality of life (HRQOL) in healthy children and adolescents and those with acute and chronic health conditions. The PedsQL Measurement Model uses a modular approach that integrates both generic core scales and disease-specific modules into one measurement system. (Copyright 1998-2008 James W. Varni, Ph.D.) 